\title{
Recognizing the Role of the Posterolateral Corner in Patients Undergoing Total Knee Arthroplasty for Fixed Varus Deformity
}

\author{
Ashok Rajgopal \\ Sumit Kumar \\ Kalpana Aggarwal
}

Institute of Musculoskeletal Disorders and Orthopaedics, Medanta- The Medicity Hospital, Gurugram, Haryana, I2200I, India
Correspondence: Ashok Rajgopal Institute of Musculoskeletal Disorders and Orthopaedics, Medanta- The Medicity Hospital, Gurugram, Haryana, |2200I, India

Tel +9l 98। I0792 I I

Email a_rajgopal@hotmail.com
Purpose: Varus deformity is the commonest presentation of the arthritic knee requiring surgical intervention. While correctable deformities lend themselves to realignment options like unicompartmental replacement, fixed deformities often need a total knee replacement. Current treatment options for patients with fixed coronal varus malalignment undergoing total knee arthroplasty include varying degrees of medial soft tissue releases, often leading to infringement of the medial collateral ligament complex and increased use of constrained options. We describe the role of the posterolateral (PL) tether in a select subgroup of patients needing release to achieve correction and minimising use of constrained options.

Patients and Methods: A total of 384 patients with fixed varus deformity were retrospectively evaluated and categorised on the basis of weight bearing $\mathrm{x}$-rays into four groups, namely, knees with angulation (F1), angulation with subluxation and torsion (F2), medial translation (F3) and deformity with major medial bone loss (F4). From this cohort, we identified patients with a tight PL tether that needed release to achieve good correction. These were predominantly in the F2 and F3 subgroups. Functional scores and outcomes were evaluated at a mean follow-up of 120.23 months.

Results: F1 cohort achieved good correction with medial soft tissue release, while F2 and F3 cohorts often needed a PL release. While functional outcomes and scores were comparable in both groups, survivorship was better in the group where release was done.

Conclusion: We recognise the role of the PL tether in a subgroup of patients with recalcitrant fixed varus deformities. Sequential release helped achieve good outcomes with minimal use of constrained options.

Level of Evidence: Three.

Keywords: varus deformity, medial release, posterolateral complex, constrained implants

\section{Introduction}

Knee Arthritis is one of the most common causes of disability in Orthopaedics. ${ }^{1}$ Advanced knee arthritis not responding to conservative management is treated by total knee arthroplasty (TKA) with excellent long-term outcomes and survivorship.

Varus malalignment accounts for the majority of knees needing intervention and in advanced cases, a TKA. ${ }^{2}$ These are knees with a hip knee ankle (HKA) angle less than $177^{\circ} .^{3}$ They need to be differentiated from a physiological varus alignment of the limb that is developmental and almost always completely asymptomatic. ${ }^{4}$

The valgus knee has been well defined with robust classification and treatment algorithms. It has been described as a triplanar deformity with adaptive changes, 
both on the femoral and tibial sides with hypoplasia of the lateral femoral condyle. ${ }^{5,6}$

In contrast, varus deformities have a paucity of literature in terms of classification. Varus knees have been typed and classified with incremental deformities of $10^{\circ}$ (Substantial $\left\{11-20^{\circ}\right\}$, Important $\left\{21-30^{\circ}\right\}$, and Extreme $\left.\left.\left\{>30^{\circ}\right\}\right)\right)^{7}$ Thienpont and Parvizi ${ }^{8}$ classified the varus knee into Type IA (intra-articular deformity), Type M (metaphyseal deformity within $5 \mathrm{~cm}$ of joint line) either at the femoral (F) or tibial ( $\mathrm{T}$ ) level and Type D (diaphyseal deformity at least $5 \mathrm{~cm}$ away from the joint line) at the tibial level, femoral level or both. Other available classifications include the Ahlback and the Kellgren-Lawrence (K-L) ${ }^{9}$ grading, which are essentially grading systems for the degree of arthritis and are radiological in nature. Typically, K-L grade 3 and 4 are candidates for TKA. These available classifications are either radiological, descriptive in terms of severity, or describe the deformity in relation to their anatomical location relative to the joint. Current available options for treatment of the varus knee include varying degrees of posteromedial soft tissue release, removal of medial and posterior femoral osteophytes, reduction osteotomy of the tibia and manipulating the distal femoral and proximal tibial cuts. ${ }^{10-12}$ Some authors recommend manipulating the medial collateral ligament (MCL) complex either by pie-crusting the $\mathrm{MCL}^{13-19}$ or performing a Medial Epicondylar Osteotomy (MEO) with good outcomes. ${ }^{20-23}$ There is limited reference in literature, to the role of the posterolateral corner (PLC) in the management of the fixed varus knee.

Recognizing this, we categorized fixed varus knees and offered a treatment algorithm that obviates the need to violate the MCL complex and thereby minimizes use of constrained implants. We believe this methodology offers an understanding of the involved contracted soft tissues in this cohort of patients.

The categorization of fixed varus knees (deformity not corrected to neutral alignment by valgus stress application) was done on the basis of clinical and radiological assessment. They were classified as F1 group -varus knees with angulation, F2 group - varus with subluxation and torsion with evidence of compromise of interosseous space, F3 group - varus and translation and F4 group having varus deformity with major bone loss.

\section{Materials and Methods}

This is a retrospective single centre study of 415 consecutive symptomatic arthritic patients with fixed varus deformity treated between 2009 and 2011. This study was undertaken after obtaining approval from Medanta Institutional Review Board. All patients with fixed coronal varus malalignment of $>10^{\circ}$ measured on weight bearing long leg radiographs were included. Patients with history of prior knee surgery, inflammatory arthritis and malunited periarticular fractures around the knee with extra articular deformities were excluded from the study.

Of the 415 patients, 26 were lost to follow-up and 5 refused enrolment in the study, leaving a final cohort of 384 patients (384 knees). An informed consent to review their medical records, was obtained from all patients. All patients underwent a standard radiological protocol which included long leg weight bearing X-Rays, Lateral and Skyline views of the patella. The protocol included obtaining a true weight bearing AP X-Ray with the patella positioned anteriorly. All radiographs were evaluated and categorized into Grades F1, F2, F3 and F4 as per our defined guidelines, shown in Figures 1A-D.

F1 - Varus with angulation. A line drawn from the lateral most part of the lateral femoral condyle does not transect the tibia (Figure 1A).

F2 - Varus with subluxation and torsion- a line drawn from the lateral most part of the lateral femoral condyle transects the tibia with evidence of compromise of the interosseous space (Figure 1B).

F3 - Medial translation of tibia relative to femur (Figure 1C).

F4 - Varus deformity with major bone loss (Figure 1D).

Of the 384 knees, 94 were Grade F1, 268 F2, 18 F3 and 4 were F4. There were 290 females and 94 males in this study. The average age was 61 years (Range 53-78), and average BMI was 30.2 (Range 26-38). All patients were clinically evaluated for range of motion (ROM), Knee Society Score (KSS), Western Ontario and McMaster Universities Osteoarthritis Index (WOMAC) score and use of walking aids. ROM was measured using a hand held long arm goniometer by a senior physiotherapist. A consistent finding in all patients with F2 and F3 deformities was the externally rotated position of the tibial tuberosity indicating a fixed external rotation torsional deformity of the tibia. Patients were followed up for a mean period of 120.23 months (SD 7.82) (Range 108132 months), initially at 3, 6 and 12 months and then annually. Statistical analysis was done using IBM SPSS 26. Standard and Mean Deviations were calculated using Descriptive Statistics and ANOVA. P-value relevance was set at 0.05 . 


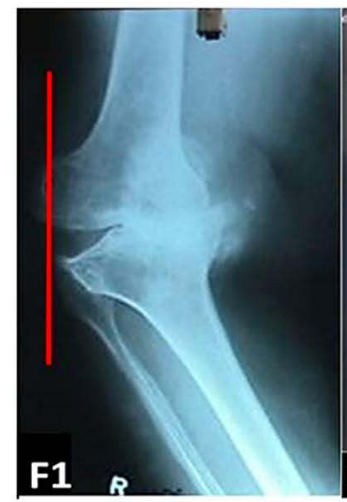

A

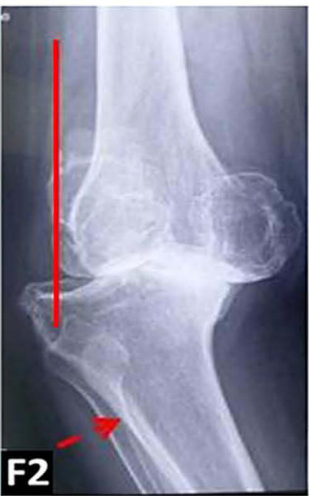

B

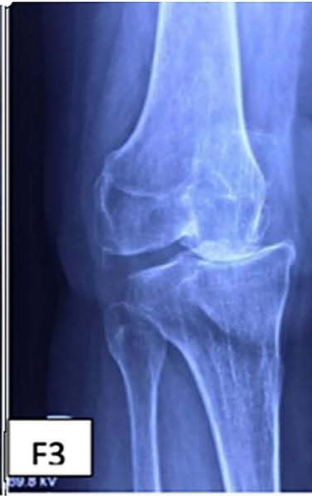

C

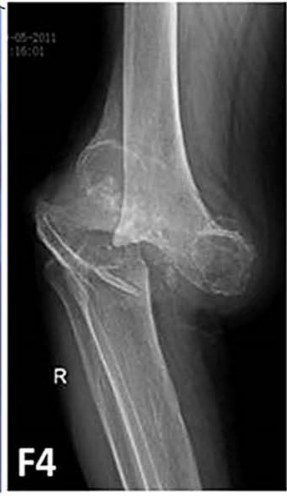

D

Figure I Categorization of radiographs as per our defined guidelines. (A) FI - Varus with angulation. A line drawn from lateral most part of lateral femoral condyle does not transect the tibia. (B) F2 - Varus with subluxation and torsion a line drawn from lateral femoral condyle transects the tibia with evidence of compromise of the interosseous space (arrow). (C) F3 - Medial translation of tibia relative to femur. (D) F4 - Varus deformity with major bone loss.

\section{Surgical Technique}

All patients were operated by the senior surgeon under tourniquet using a midline skin incision and a medial parapatellar approach. A wide posteromedial soft tissue release was done extending up to the semimembranosus insertion. Peripheral osteophytes were removed from the medial proximal tibia and femur. Adequacy of correction was checked sequentially. The tibia was cut at right angles to the mechanical axis and the distal femur resected at a $5^{\circ}$ distal valgus resection angle. External rotation was set at $3^{\circ}$ in relation to the posterior condylar line. Posterior femoral osteophytes were removed. Anterior referencing instruments were used, offsetting the issue of posteromedial femoral overgrowth and its consequent tendency to internally rotate the femoral component. ${ }^{24}$ In severe deformities tibial resection was limited to 6-7 $\mathrm{mm}$ and reduction osteotomy carried out where needed. This technique allowed excellent correction of all F1 cohort of knees. The posterior cruciate ligament (PCL) was retained or sacrificed depending on the severity of the deformity and the presence of intercondylar osteophytes.

In the F2 and F3 cohort of patients however, despite the soft tissue release described, varus subluxation and torsion were seen to be recalcitrant in a significant number of cases. Distracting the knee to check restoration of a symmetrical rectangular flexion and extension gap using a lamellar spreader revealed a tight band in the posterolateral corner that prevented achieving this balance. This comprised the posterolateral capsule and the popliteus tendon. The posterolateral capsule was released using electrocautery at the level of the resected tibia while the popliteus tendon was pie crusted using 18 gauge needle, as shown in Figure 2. This corrected the torsion and subluxation, satisfactorily aligning the components. The radiological demonstration of this release is shown in Figure 3.

This posterolateral release differs from a similar release done for the valgus knee in that the iliotibial band, lateral retinaculum and the lateral collateral ligament are left untouched. In our study, posterolateral release was

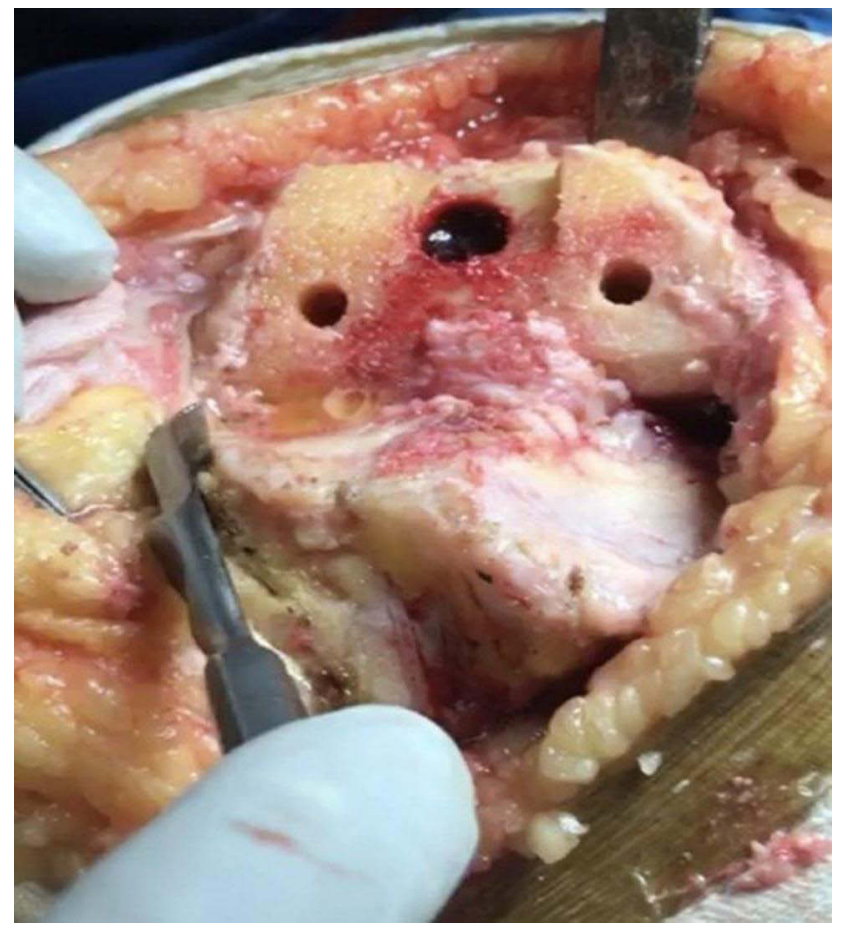

Figure 2 Intra-operative image showing release of posterolateral corner. 

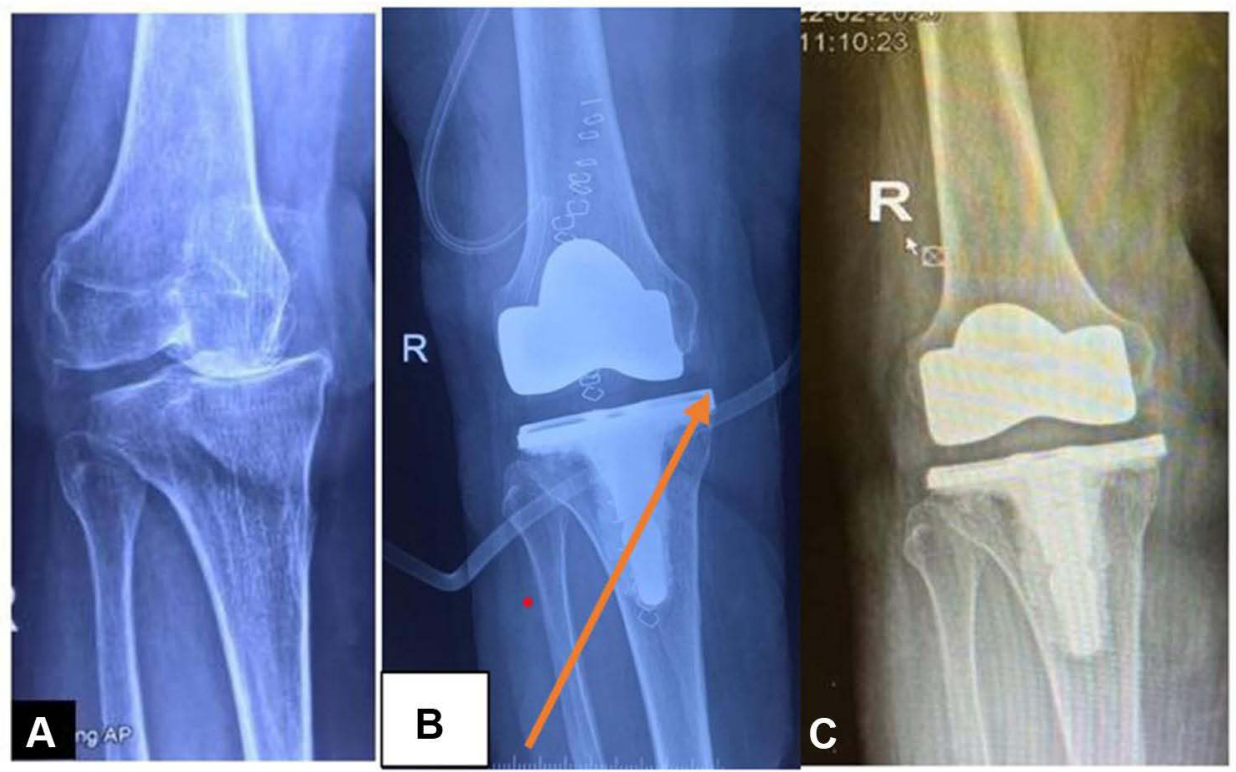

Figure 3 X-ray images before and after PL release. (A) Preoperative X-Ray image showing medial translation. (B) Post operative X-ray image without PL release-showing persistent subluxation. (C) Post operative X-ray image After PL release with restoration of alignment.

needed in 177 of 268 cases of Grade F2 (66\%) and all 18 cases of Grade F3 (100\%) varus knees.

All implants in this study were cemented with selective resurfacing of the patella in cases with severe degenerative patellar changes.

\section{Results}

The mean KSS, WOMAC score and the ROM improved considerably from the pre-operative status across all groups as explained in Table 1. The mean preoperative varus malalignment was $28^{\circ}\left(10-45^{\circ}\right)$ which was corrected post operatively to a mean of $2^{\circ}$ valgus $\left(-2\right.$ to $\left.4^{\circ}\right)$.

The mean ROM improved significantly from a preoperative mean of $86.13^{\circ}$ (SD 13.21) (range 65.00$92.65^{\circ}$ ) to a post-operative mean of $119.20^{\circ}$ (SD 11.99) (range 93.75 to $126.22^{\circ}$ ) at the time of final follow up, in all four cohorts of patients. The KSS of all the cohorts improved significantly from a preoperative mean of 29.88 (SD 8.56) to 84.54 (SD 5.98) postoperatively, at the time of final follow up. The WOMAC scores of the four groups of patients similarly improved significantly from a preoperative mean score of 73.54 (SD 7.39) to a postoperative mean of 15.86 (SD 3.91) at the time of final follow up across all four cohorts.

Primary implants (cruciate retaining or posterior stabilized) were used in 92 of 94 in Grade F1, 244 of 268 in Grade F2, and 13 of 18 in Grade F3 knees. All patients in the F4 cohort needed constrained knee implants.
Patients were regularly followed up with AP and Lateral X-Rays at each follow up.

Radiolucent lines were seen in 34 knees. They were present most often in the tibia, zone 1 and 2 (26 knees) and zone 1 in femur ( 8 knees). The radiolucency was non progressive in 25 knees on the tibial side and 7 knees on the femoral side. Progressive radiolucency in the tibia was seen in 4 knees in Grade F2 cohort, which went on to develop aseptic loosening needing subsequent revision.

Table I Statistical Analysis of ROM, KSS and WOMAC Score Across All 4 Groups

\begin{tabular}{|c|c|c|c|c|}
\hline Variables & Grades & Pre Op with SD & Post Op with SD & p-value \\
\hline \multirow[t]{5}{*}{ ROM } & $\mathrm{FI}$ & 92.65 (11.72) & $126.22(9.38)$ & 0.000 \\
\hline & F2 & 85.54 (II.43) & $118.64(9.05)$ & 0.000 \\
\hline & F3 & $67.77(14.16)$ & $100.33(10.43)$ & 0.000 \\
\hline & F4 & $65.00(8.16)$ & 93.75 (16.52) & 0.000 \\
\hline & Total & $86.13(13.21)$ & 119.20 (11.99) & 0.000 \\
\hline \multirow[t]{5}{*}{ KSS } & $\mathrm{FI}$ & $34.54(7.35)$ & $87.72(4.53)$ & 0.000 \\
\hline & F2 & $28.98(7.78)$ & 84.24 (5.29) & 0.000 \\
\hline & F3 & $23.66(8.60)$ & $75.94(5.68)$ & 0.000 \\
\hline & F4 & $16.00(2.94)$ & $74.25(4.50)$ & 0.001 \\
\hline & Total & $29.88(8.56)$ & $84.54(5.98)$ & 0.000 \\
\hline \multirow[t]{5}{*}{ WOMAC } & $\mathrm{FI}$ & 71.69 (6.72) & $13.98(2.92)$ & 0.000 \\
\hline & F2 & $73.50(7.17)$ & $16.15(3.66)$ & 0.000 \\
\hline & F3 & 81.11 (7.88) & $19.22(4.18)$ & 0.000 \\
\hline & $\mathrm{F} 4$ & $83.25(6.55)$ & $21.75(2.75)$ & 0.000 \\
\hline & Total & 73.54 (7.39) & $15.86(3.91)$ & 0.000 \\
\hline
\end{tabular}


Table 2 Statistical Analysis of F2 Group

\begin{tabular}{|l|r|r|r|r|}
\hline F2 & Total N & N of Events (Revisions) & \multicolumn{2}{|c|}{ Censored } \\
\cline { 3 - 5 } & & & N & \multicolumn{2}{|c|}{ Percent } \\
\hline Released & 177 & 2 & 175 & $98.9 \%$ \\
Not Released & 91 & 7 & 84 & $92.3 \%$ \\
Overall & 268 & 9 & 259 & $96.6 \%$ \\
\hline
\end{tabular}

A total of 16 patients (4.2\%) developed complications needing reoperations. There were 3 cases of deep infection requiring a two stage revision, 2 patients sustained a periprosthetic fracture and 2 patients had an extensor mechanism disruption requiring surgical intervention. In addition, there were 4 cases of aseptic loosening, 1 case of arthrofibrosis, and 4 patients with cruciate retaining (CR) implants presented with late instability due to PCL rupture. Of the 9 patients who underwent revision surgery for aseptic loosening and arthrofibrosis and instability in the F2 cohort, 3 patients had a PLC release during the primary surgery, while 6 did not.

A total of 31 knees (all unresurfaced patellae) had painless patellar crepitus which however did not require any intervention. In our evaluations, across all variables measured, the least improvement was seen in F3 and F4 cohorts.

\section{Statistical Analysis}

In the F2 cohort, survivorship in the group where PLC release was done was $98.9 \%$ (175/177 patients) and 92.3\% (84/91 patients) where PLC release was not done as described in Table 2 . This difference is statistically significant with a $\mathrm{p}$ value of 0.004 . Overall survivorship of the F2 cohort at final follow up was $96.6 \%$.

In the F2 cohort, of the 177 patients where PLC release was done, 8 (4.5\%) patients had poor functional outcomes in terms of ROM and functional scores as compared to 11 of 91 (12.1\%) patients where PLC release was not done. Odds ratio for lower KSS was 2.9 in the non-released group as compared to the released group. The power was calculated for ROM, KSS, and WOMAC, based on these results the implicit power of the study to detect the difference as significant is $100.0 \%$ with confidence level $95 \%$ as discussed in Table 3 .

\section{Discussion}

Varus deformities account for over $90 \%$ of all cases undergoing knee replacement surgery for the arthritic knee. ${ }^{2}$ Various treatment algorithms have been described to treat the fixed varus deformity, with good to excellent results. While most of these approaches recommend releasing the
Table 3 Power Analysis of ROM, KSS and WOMAC Score

\begin{tabular}{|l|c|c|c|c|}
\hline Variables & Grades & $\begin{array}{c}\text { Pre Op and } \\
\text { SD }\end{array}$ & $\begin{array}{c}\text { Post Op and } \\
\text { SD }\end{array}$ & Power \\
\hline ROM & FI & $92.65(11.72)$ & $126.22(9.38)$ & 100.0 \\
& F2 & $85.54(12.60)$ & $118.64(11.52)$ & 100.0 \\
& F3 & $67.77(14.16)$ & $100.33(10.43)$ & 100.0 \\
& F4 & $65.00(8.16)$ & $93.75(16.52)$ & 100.0 \\
& Total & $86.13(13.21)$ & $119.20(11.99)$ & 100.0 \\
\hline \multirow{2}{*}{ KSS } & FI & $34.54(7.35)$ & $87.72(4.53)$ & 100.0 \\
& F2 & $28.9(11.0)$ & $84.2(7.51)$ & 100.0 \\
& F3 & $23.66(8.60)$ & $75.94(5.68)$ & 100.0 \\
& F4 & $16.00(2.94)$ & $74.25(4.50)$ & 100.0 \\
& Total & $29.88(8.56)$ & $84.54(5.98)$ & 100.0 \\
\hline \multirow{2}{*}{ WOMAC } & FI & $71.69(6.72)$ & $13.98(2.92)$ & 100.0 \\
& F2 & $73.50(10.15)$ & $16.16(5.20)$ & 100.0 \\
& F3 & $81.11(7.88)$ & $19.22(4.18)$ & 100.0 \\
& F4 & $83.25(6.55)$ & $21.75(2.75)$ & 100.0 \\
& Total & $73.54(7.39)$ & $15.86(3.91)$ & 100.0 \\
\hline
\end{tabular}

deep MCL up to the semimembranosus attachment, ${ }^{10}$ some recommend downsizing and lateralising the tibial component and performing a reduction osteotomy to achieve correction. ${ }^{12}$ Other authors recommend releasing the distal tibial attachment of the superficial MCL, ${ }^{10,12}$ or fractional lengthening of the MCL by pie-crusting the $\mathrm{MCL}^{13-16}$ or performing a medial epicondylar osteotomy. ${ }^{6,20}$

Given the multiple available options that often infringe the "Critical MCL Complex" with increased use of constrained options, the authors segregated different categories of varus knees, based on their radiological appearance, clinical presentation and soft tissue involvement and proposed a treatment algorithm. Both the categorization and consequent treatment algorithm are based on anatomical factors and contracted soft tissues, recognizing the role of the tight posterolateral corner, in a select cohort of fixed varus knees. The authors observed the presence of a fixed externally rotated position of the tibial tuberosity in these patients.

These were the knees with subluxation and torsion (Grade F2), and the varus translational cohort (Grade 
F3). In both these groups of patients, limiting the tibial resection to $6-7 \mathrm{~mm}$, recognizing, identifying and releasing the "posterolateral tether" helped restore alignment, balanced the knee and provided for a stable articulation. More importantly it did not infringe the integrity of the "MCL Complex" thereby preserving the crucial medial stabilizer of the knee and reducing the need for "constrained implant options".

Rahm et al $^{22}$ compared a cohort of three groups of patients with severe varus knees treated by manual instrumentation, computer navigation and patient-specific instrumentation. They reported the least outliers in the patient-specific cohort and recommended this as their preferred option.

While Bellemans et $\mathrm{a}^{15}$ recommended pie-crusting the MCL using a 19 gauge needle for controlled release, Yagishita et $\mathrm{al}^{16}$ reported an uncontrolled opening of more than $35 \mathrm{~mm}$ in 2 of the 5 cases using this option. Verdonk et $\mathrm{al}^{14}$ recommended pie-crusting the MCL, release of the superficial MCL, and in addition releasing the semimembranosus in $63 \%$ of the cases. Kwak et a ${ }^{17}$ reported unpredictable gap opening and early over release in $70 \%$ of cases specially when releases were done in flexion. Gap measurement increments of 1-3 $\mathrm{mm}$ in extension and $2-6 \mathrm{~mm}$ in flexion were reported. Meneghini et $\mathrm{al}^{18}$ also questioned the safety of the pie-crusting technique, observing a "stair-step" failure at the joint line.

Engh and Ammeen, ${ }^{20}$ Ranawat et al, ${ }^{6}$ and $\mathrm{Stan}^{23}$ recommended a MEO to correct the deformity and balance the MCL. Despite a high incidence of fibrous union of the osteotomy these authors did not report any adverse outcomes. Mihalko et al ${ }^{19,21}$ questioned the need for performing a MEO reporting increased coronal and transverse plane laxity at $60^{\circ}$ and $90^{\circ}$ of flexion and a greater need for constrained implants.

Several studies ${ }^{25,26}$ have described the role of PLC release in improving the tight lateral flexion gap (often seen in Grade F2 and F3 deformities). In severe varus arthritic knees external torsion of the proximal tibia is observed. $^{27}$ Long standing external torsion may be a possible aetiological cause for the contracted posterolateral structures. ${ }^{28}$ The complicated anatomy of the PLC includes the lateral collateral ligament (LCL), the popliteus muscle tendon ligament unit (PMTL), the fabellofibular ligament, the arcuate ligament and the posterolateral capsule. ${ }^{29}$ No individual structure in isolation is a primary restraint for instability and releasing the PLC alone does not lead to instability of the knee. ${ }^{28,30}$ Releasing the tight
PLC at the resected level of the tibia allows it to move forward, correcting the lateral translational and rotational deformity. ${ }^{27}$

Using this algorithmic approach, we achieved good correction of the fixed varus arthritic knee without violating the MCL complex and considerably minimizing the use of constrained (Rotating Hinge Knee and Legacy Constrained Condylar Knee) articulations with excellent long-term survivorship. This study demonstrates good outcomes and survivorship in the cohort where posterolateral corner release was done.

\section{Limitations}

Limitations of this article include its retrospective nature and challenges in getting standardized long leg weight bearing X-Rays in patients having varus and flexion deformity with associated torsion. Associated foot deformities may also have affected accurate measurements. Another limitation of this study is that no biomechanical evaluation of the knees was done. Undertaking this may possibly have extended our understanding of this anatomical structure.

\section{Conclusion}

Fixed varus arthritic knees require various medial and posteromedial soft tissue releases, which may compromise the medial ligament complex leading to use of additional constrained articulation. We present our understanding of this cohort of patients, recognising the role of the posterolateral tether in a select subgroup of patients with fixed varus deformity. Identifying this tether and its consequent release helps in achieving a well balanced and aligned knee without infringing the MCL complex. In our experience using this approach we were able to considerably reduce the need for constrained options with excellent long-term outcomes and survivorship.

\section{Disclosure}

The authors report no conflicts of interest in this work.

\section{References}

1. Hayami T. Osteoarthritis of the knee joint as a cause of musculoskeletal ambulation disability symptom complex (MADS). Clin Calcium. 2008; 18:1574-1580.

2. Chou PH, Chen WM, Chen CF, et al. Clinical comparison of valgus and varus deformities in primary total knee arthroplasty following midvastus approach. $J$ Arthroplasty. 2012;4:604. doi:10.1016/j. arth.2011.06.015 
3. Moreland JR, Bassett LW, Hanker GJ. Radiographic analysis of the axial alignment of the lower extremity. J Bone Joint Surg Am. 1987;69:745. doi:10.2106/00004623-198769050-00016

4. Nam D, Shah RR, Nunley RM, et al. Evaluation of the 3-dimensional weight bearing orientation of the normal adult knee. $J$ Arthroplasty. 2014;29(5):906. doi:10.1016/j.arth.2013.10.024

5. Krackow KA, Jones MM, Teeny SM, Hungerford DS. Primary total knee arthroplasty in patients with fixed valgus deformity. Clin Orthop Relat Res. 1991;273:9-18.

6. Ranawat AS, Ranawat CS, Elkus M, Rasquinja VJ, Rossi R, Babhulkar S. Total knee arthroplasty for severe valgus deformity. J Bone Joint Surg Am. 2005;87(Supp11; Pt. 2):272-284.

7. De Muylder J, Victor J, Cornu O, Kaminski L, Thienpont E. Total knee arthroplasty in patients with substantial deformities using primary knee components. Knee Surg Sports Traumatol Arthrosc. 2015;23(12):3653-3659. doi:10.1007/s00167-014-3269-x

8. Thienpont E, Parvizi J. A new classification for the varus knee. J Arthroplasty. 2016;31(10):2156-2160. doi:10.1016/j.arth.2016.03.034

9. Menkes CJ. Radiographic criteria for classification of osteoarthritis. J Rheumatol Suppl. 1991;27:13.

10. Laskin RS, Schob CJ. Medial capsular recession for severe varus deformities. J Arthroplasty. 1987;2:313. doi:10.1016/S0883-5403(87) 80065-7

11. Engh GA. The difficult knee. Severe varus and valgus. Clin Orthop. 2003;416:58. doi:10.1097/01.blo.0000092987.12414.fc

12. Dixon MC, Parsch D, Brown RR, et al. The correction of severe varus deformity in total knee arthroplasty by tibial component downsizing and resection of uncapped proximal medial bone. J Arthroplasty. 2004;19(1):19. doi:10.1016/j.arth.2003.08.001

13. Meftah M, Blum YC, Raja D, Ranawat AS, Ranawat CS. Correcting fixed varus deformity with flexion contracture during total knee arthroplasty: the "inside-out" technique: AAOS exhibit selection. J Bone Joint Surg Am. 2012;94(10):e66. doi:10.2106/JBJS.K.01444

14. Verdonk PC, Pernin J, Pinaroli A, Selmi AS, Neyret P. Soft tissue balancing in varus total knee arthroplasty: an algorithmic approach Knee Surg Sports Traumatol Arthrosc. 2009;17(6):660-666. doi:10.1007/s00167-009-0755-7

15. Bellemans J, Vandenneucker H, Van Lauwe J, Victor J. A new surgical technique for medial collateral ligament balancing: multiple needle puncturing. $J$ Arthroplasty. 2010;25(7):1151-1156. doi:10.1016/j. arth.2010.03.007

16. Yagishita K, Muneta T, Ikeda H. Step-by-step measurements of soft tissue balancing during total knee arthroplasty for patients with varus knees. J Arthroplasty. 2003;18(3):313-320. doi:10.1054/arth.2003.50088

17. Kwak DS, In Y, Kim TK, Hs C, Koh IJ. The pie-crusting technique using a blade knife for medial collateral ligament release is unreliable in varus total knee arthroplasty. Knee Surg Sports Traumatol Arthrosc. 2016;24(1):188-194. doi:10.1007/s00167-014-3362-1
18. Meneghini RM, Daluga AT, Sturgis LA, Lieberman JR. Is the pie-crusting technique safe for MCL release in varus deformity correction in total knee arthroplasty? J Arthroplasty. 2013;28 (8):1306-1309. doi:10.1016/j.arth.2013.04.002

19. Mihalko WM, Woodard EL, Hebert CT, Crockarell JR, Williams JL. Biomechanical validation of medial pie-crusting for soft tissue balancing in knee arthroplasty. J Arthroplasty. 2015;30(2):296-299. doi:10.1016/j.arth.2014.09.005

20. Engh GA, Ammeen D. Results of total knee arthroplasty with medial epicondylar osteotomy to correct varus deformity. Clin Orthop Relat Res. 1999;367:141-148. doi:10.1097/00003086-199910000-00017

21. Mihalko WM, Saeki K, Whiteside LA. Effect of medial epicondylar osteotomy on soft tissue balancing in total knee arthroplasty. Orthopedics. 2013;36:e1353-e1357. doi:10.3928/0147744720131021-14

22. Rahm S, Camenzind RS, Hingsammer A, et al. Postoperative alignment of TKA in patients with severe preoperative varus or valgus deformity: is there a difference between surgical technique? $B M C$ Musculoskelet Disord. 2017;18:272. doi:10.1186/s12891-017-1628-8

23. Stan G. Medial edpicondyle osteotomy for balancing severe varus knee. In: Knee Surgery Reconstruction and Replacement. IntechOpen; 2019. doi:10.5772/intechopen.89740

24. Choudhary P, Bahre S, Tantuway V, et al. Anterior referencing versus posterior referencing in total knee arthroplasty: a prospective observational study. Int J Res Orthop. 2017;3(1):66-70. doi:10.18203/ issn.2455-4510.IntJResOrthop20164789

25. Peters CL, Mohr RA, Bachus KN. Primary total knee arthroplasty in the valgus knee: creating a balanced soft tissue envelope. J Arthroplasty. 2001;16:721-729.

26. Matsueda M, Gengerke TR, Murphy M, Lew WD, Gustilo RB. Soft tissue release in the total knee arthroplasty. Cadaver study using knees without deformities. Clin Orthop Relat Res. 1999;366:264-273. doi:10.1097/00003086-199909000-00034

27. Crane L. Femoral torsion and its relation to toeing-in and toeing-out. J Bone Joint Surg Am. 1959;41-A(3):421e8.

28. Peters CL, Severson E, Crofoot C, Allen B, Erickson J. Popliteus tendon release in the varus or neutral knee: prevalence and potential etiology. J Bone Joint Surg Am. 2008;90(Suppl 4):40-46. doi:10.2106/JBJS.H.00687

29. Sudasna S, Harnisiriwattanagit K. The ligamentous structures of the posterolateral aspect of the knee. Bull Hosp Jt Dis Orthop Inst. 1990;50:35-40.

30. Pasque C, Noyes FR, Gibbons M, Levy M, Grood E. The role of the popliteofibular ligament and the tendon of popliteus in providing stability in the human knee. J Bone Joint Surg. 2003;85-B:292-298. doi:10.1302/0301-620X.85B2.12857
Orthopedic Research and Reviews

\section{Publish your work in this journal}

Orthopedic Research and Reviews is an international, peer-reviewed, open access journal that focusing on the patho-physiology of the musculoskeletal system, trauma, surgery and other corrective interventions to restore mobility and function. Advances in new technologies, materials, techniques and pharmacological agents are particularly welcome. The manuscript management system is completely online and includes a very quick and fair peer-review system, which is all easy to use. Visit http://www.dovepress.com/testimonials.php to read real quotes from published authors. 